\title{
SILICON AND HYBRID MICRO-ELECTRONIC SENSORS
}

\author{
S. MIDDELHOEK, D.J.W. NOORLAG and G.K. STEENVOORDEN \\ Delft University of Technology, Delft, The Netherlands
}

(In final form April 23, 1982)

The penetration of micro-electronics into new markets is seriously hampered by the lack of efficient, low-cost sensors. It is not surprising therefore to find that these devices are the subject of research in many laboratories. This paper gives a review of the most important sensors which are fabricated in silicon planar or hybrid technology. The review is preceded by some general considerations with respect to measurement systems and signal conversion.

\section{INTRODUCTION}

Information-processing systems consist of a central signal processor plus input and output transducers which interface to the non-electronic environment (Figure 1). In the input transducer -often called sensor- a measurand such as temperature, displacement, concentration, switch position, etc., is converted into an electronic signal. In the signal processor - often called modifier - this electronic signal is in some way modified, e.g. amplified, filtered or converted from an analog to a digital signal. In the output transducer either the electronic signal is again converted into a signal which can be perceived by one of our senses or it is used to cause some action, e.g. to print a character or to close a valve. ${ }^{1}$ Until recently the signal processor was more or less the only part of the informationprocessing system that was the target of very intensive and costly research and development work.

As a result of this activity we have at present at our disposal a large number of very sophisticated micro-electronic building blocks such as microprocessors, microcomputers, A/D converters, voltage/frequency converters, operational amplifiers, etc. All these components are characterized by an unprecendented performance/price ratio.

However, the neglect of the transducer side of the system has now caused an undesirable situation marked by the fact that the further penetration of micro-electronics into the many newly envisaged markets is not yet possible because of the lack of transducers with performance/price ratios comparable to those of the micro-electronic circuits $^{2}$ and without problems in interfacing with the electronic modifier.

Understandably, the transducer field is currently attracting more and more interest, so that in many laboratories throughout the world new transducer principles and structures are bieng studied.

The aim of this paper is to give a review of the most important sensors as described in the literature. The great progress in silicon integrated circuits during recent decades has been made possible by the invention of the silicon planar technology (photolithography, diffusion, epitaxy and metallization). Not surprisingly attempts are being made by the electronics industry and university laboratories to extent the application of this successful technology to sensors as well. Also through thick- and thin-film technology a large number of useful and innovative sensors have been fabricated in recent decades. One section of this paper (Section 3) will be devoted to a discussion of the so-called silicon micro-transducers (SMT's) and another (Section 4) to the so-called hybrid transducers. 


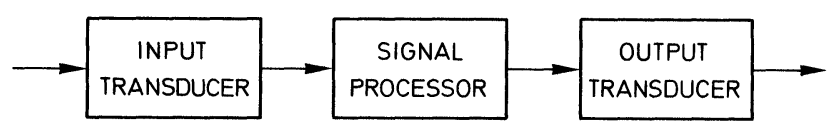

FIGURE 1 Block diagram of a measurement or control system.

But first we will turn our attention, in Section 2, to some considerations with respect to the different signals which have to be detected by the sensors.

\section{SIGNAL CONVERSION}

In an input transducer or sensor a measurand such as temperature, pressure or $\mathrm{pH}$ value is converted into an electronic signal. Because information flow is inconceivable without the flow of energy, sensors are in fact devices that convert energy from one form into another. For instrumentation purposes six energy and thus six signal domains can be distinguished: radiant signals (light intensity, phase), mechanical signals (pressure, displacement), thermal signals (temperature, heat), electrical signals (voltage, resistance), magnetic signals (field strength, permeability) and chemical signals (concentration, toxicity). ${ }^{3}$

Figure 2 shows a diagram indicating the five signal conversions which can occur in sensors. In a signal processor (the central building block of an information-processing system) the signal remains in the electrical signal domain, whereas in the output transducer an electrical signal is converted back into one of the other five signal domains. For example, in case of an optical display the signal is converted into the radiant signal domain.



FIGURE 2 Diagram indicating the five possible signal conversions in sensors. 
In order to convert signals an immense number of physical effects are available and have been described in the literature on solid-state physics. For instance to convert a radiant signal into an electrical signal, such effects as the photovoltaic effect, photoconductance, photodielectric effect, photomagneto-electric effect and the Dember effect can be used. Two kinds of sensors, based on these effects, can be distinguished. Bear in mind that some sensors generate an electrical output without an auxiliary energy source and others only function when auxiliary energy is supplied.

By restricting ourselves to sensors in the radiant signal domain, we can use the photovoltaic effect to make sensors of the former kind. Without an auxiliary energy source a light intensity is converted into a voltage. The solar cell is based on this effect. Sensors operating without auxiliary sources are called self-generating sensors.

The second kind of sensor is known under the name of modulating sensor. When, for instance, a photoconductor or a photodiode is exposed to light, electron-hole pairs are generated, so that the resistance of the device decreases. This decrease can be detected by using an auxiliary energy source. In Figure 3 some physical effects which can be used for self-generating and modulating sensors are tabulated for the five different signal domains.

\section{SILICON MICRO-TRANSDUCERS (SMT'S)}

The silicon planar technology makes it possible to batch fabricate large numbers of identical circuits, which themselves consist of a larger number of components. The three most significant features of the technology are (1) that $\mathrm{SiO}_{2}$ layers, simply obtained by oxidizing the wafer in an oxygen-containing atmosphere, can be used to mask the impurity doping process, (2) that all structures are obtained by means of photolithographic techniques and (3) that all passive and active components and their metal interconnections are obtained only by processing the silicon wafer on one side.

When the same silicon planar technology is applied to sensors, sometimes a few alterations have to be applied with respect to wafer or chip size, chip thickness, metalization, bonding, etc. ${ }^{4}$

\begin{tabular}{|c|c|c|}
\hline \multirow{2}{*}{$\begin{array}{l}\text { SIGNAL } \\
\text { DOMAINS }\end{array}$} & \multicolumn{2}{|c|}{ PHYSICAL EFFECTS } \\
\hline & SELF - GENERATING & MODULATING \\
\hline RADIANT & $\begin{array}{c}\text { PHOTOVOLTAIC } \\
\text { EFFECT }\end{array}$ & PHOTOCONDUCTANCE \\
\hline MECHANICAL & $\begin{array}{l}\text { PIEZOELECTRIC } \\
\text { EFFECT }\end{array}$ & PIEZORES ISTANCE \\
\hline THERMAL & SEEBECK EFFECT & THERMORES ISTANCE \\
\hline MAGNETIC & $\begin{array}{l}\text { MAGNETOELECTRO } \\
\text { EFFECT }\end{array}$ & MAGNETORES I S TANCE \\
\hline CHEMICAL & $\begin{array}{c}\text { CHEMO - VOLTAIC } \\
\text { EFFECT }\end{array}$ & IONCONDUCTANCE \\
\hline
\end{tabular}

FIGURE 3 Physical effects for self-generating and modulating sensors. 
The most serious problems are encountered when the sensors have to be used in a nonencapsulated form. Sensors for chemical signals in particular show that this technical problem still demands ample attention. The initial steps for applying silicon planar technology to the sensor field were taken in about 1970. A few very interesting devices were the result of this effort. Nevertheless, only those devices which were designed for sensoring optical signals were widely accepted on the electronics market. Because low-cost, sophisticated signal processors, e.g. microprocessors, did not yet exist, the development of sensors for the other signal domains was slowed down or even interrupted.

Today the situation is different. Low-cost microprocessors and microcomputers are available on a large scale and much interest has been kindled in applying these components to new markets. Therefore the need today for equally low-cost sensors is very acute. This is best illustrated by the automotive industry. Many sensor technologies can be adapted in such a way that they produce suitable sensors compatible to microprocessors. In the rest of this section a number of devices which are fabricated by means of the silicon planar technology will be presented.

\subsection{SMT's for Radiant Signals}

Radiant signals embrace the whole spectrum of electromagnetic waves. This spectrum includes, in order of increasing wavelength, gamma rays, X-rays, light waves and radio waves in a range roughly between $10^{-14} \mathrm{~m}$ and $10^{4} \mathrm{~m}$. Light only represents a small portion of this spectrum from ultraviolet $\left(10^{-7} \mathrm{~m}\right)$ via visible $\left(3 \times 10^{-7} \mathrm{~m}-7 \times 10^{-7} \mathrm{~m}\right)$ to infrared $\left(10^{-4} \mathrm{~m}\right)$. For detecting visible light silicon devices have been used on a great scale during the last two decades. These devices are amply described and reviewed in literature..$^{5,6}$

Silicon with a bandgap of $1.1 \mathrm{eV}$ shows photoconductivity between $400 \mathrm{~nm}$ and 1150 $\mathrm{nm}$. A photoconductor can easily be fabricated by diffusing a p-type strip into an n-type substrate and by providing the strip with ohmic contacts at the ends. Another method is to use pn-junctions with either forward or reverse bias. When a junction is exposed to light photons create electron-hole pairs in and near the depletion layer. Because of the electric field in the junction, the electrons go towards the n-type region and the holes towards the p-type region. The result of this separation of charge carriers is that a potential difference is created across the junction, which can be externally measured and which is a measure of the incident light intensity.

When a reverse voltage is applied to a pn-junction only a very small, so-called dark current can be observed. Free charge carriers are created when the junction is exposed to light, and this raises the reverse current roughly proportional to the light intensity. A more efficient photodiode is obtained when a layer of intrinsic silicon is used between the p-type and n-type regions. Such a photodiode is known as a pin photodiode.

Sensitivities to wavelengths below $300 \mathrm{~nm}$ can be obtained by Schottky barrier goldsilicon photodiodes. In an avalanche photodiode reverse voltage breakdown and carrier multiplication is used to increase the sensitivity. In a phototransistor the basecollector junction is exposed to light. When the base is not connected the increase of the reverse current through this junction is amplified, resulting in a large collector-current change.

MOSFET's can also be used as light detectors.

Because they are both fabricated in silicon with planar silicon technology, optoelectronic devices and electronic signal-processing circuits can easily be integrated on the same chip. A large number of such integrated structures, sometimes called "smart sensors", are described in the literature. Optoelectronic silicon devices are fabricated for line and matrix scanning purposes or are combined with circuits such as Schmitt Triggers, Operational amplifiers, Darlington stages, etc. 


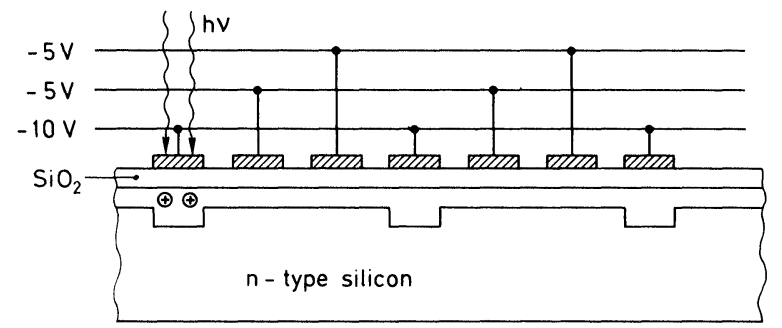

FIGURE 4 Schematic representation of a three-phase charge-coupled device (CCD).

Around 1970 a very innovative optoelectronic device called a Charge Coupled Device (CCD) was proposed. This device consists of arrays of photosensitive MOS capacitors in which electrical charge packets are stored (Figure 4). The charge can be created by lightinduced electron-hole pair formation, the number of charge carriers being a measure of the incident light intensity. By applying voltages to the electrodes in a proper sequence, the charge packets can be shifted through the semi-conductor. With CCD's it is possible to construct all solid-state linear and area optical imaging devices. CCD arrays with more than $10^{6}$ picture elements have been processed. Nevertheless, at present it is still difficult to predict whether the existing TV camera tubes will be replaced in the future by CCD devices.

\subsection{SMT's for Mechanical Signals}

A great need exists (e.g. in the automotive industry) for transducers that can measure mechanical signals such as: pressure, torque, liquid flow, thickness, level, position, velocity, acceleration, tilt, etc. The scientific literature contains several interesting descriptions of silicon devices.

a) Pressure sensor. Silicon is not piezoelectric, but shows a large piezoresistive effect. A pressure sensor making use of this effect was first described by Gieles. ${ }^{7}$ That device consists of a very thin $(15 \mu \mathrm{m}) \mathrm{n}$-type silicon pressure diaphragm with a diameter of $1 \mathrm{~mm}$ in which a Wheatstone bridge of four p-doped resistors is diffused. Devices can be made for the pressure range of 1-100 bar.

b) Accelerometer. A silicon accelerometer ${ }^{8}$ is described which consists of a very thin $(\leqslant 15 \mu \mathrm{m})$ silicon cantilever with a $200 \mu \mathrm{m}$-thick silicon mass, which serves as an inertial reference. A piezoresistive strain gauge is diffused into the cantilever. Its resistance is a measure for the acceleration of the structure. The whole structure is made in silicon with a V-groove etching technique.

c) Position detector. By using a laser, a mirror attached to the object whose position has to be measured, and a specially designed photodetector, one can measure position. ${ }^{9}$ The photodetector consists of an $8 \times 6 \mathrm{~mm}^{2}$ rectangular pn-diode (Figure 5). Contact strips as shown are provided to both p-type and n-type layers. The pn-diode is reversebiased at about $10 \mathrm{~V}$. When the laser light-spot reflected via the mirror is incident to the device, electron-hole pairs are generated at the light-spot position. In the structure shown the photocurrent will be divided between the contacts in proportion to the conductivity between the light-spot position and the contacts. The X- and Y-signals are exactly linear to the coordinates of the light-spot. Linearity is better than $0.5 \%$ full scale and resolution is about $5 \mu \mathrm{m}$. The photodetector can be made much larger. However, the device requires the diffusion depth and the impurity concentration to be the same in the whole detector, which requires careful processing. 


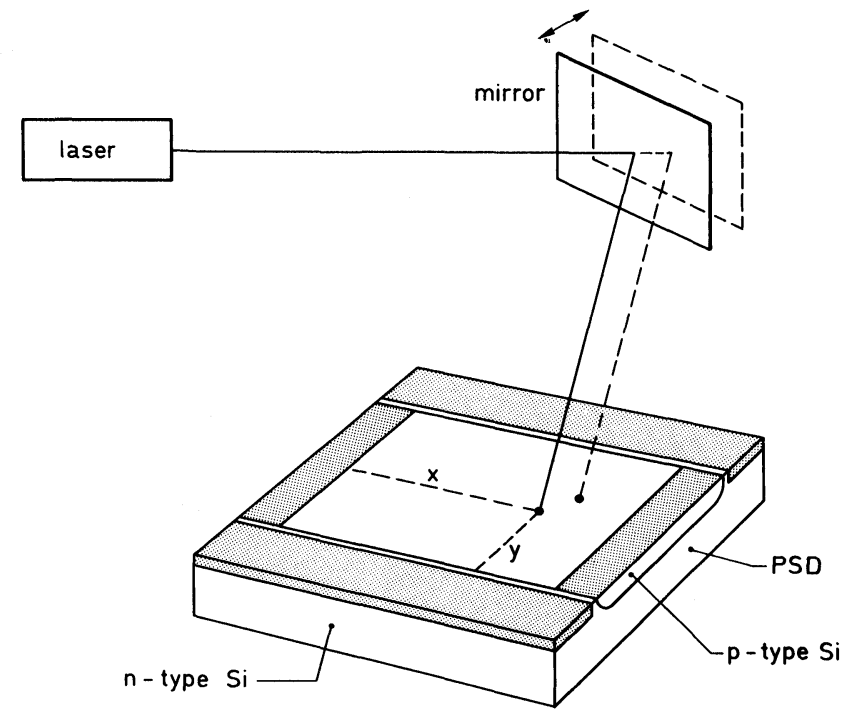

FIGURE 5 Light-spot position-sensitive detector (PSD).

d) Flowmeter. A very important physical quantity in the mechanical signal domain is gas or liquid flow. Many principles for measuring flow have been investigated in past years. A principle based on measuring temperature differences ${ }^{10}$ is suitable for realization by silicon planar technology. Three transistors are integrated on a silicon chip of $2 \times 2 \mathrm{~mm}^{2}$ (Figure 6). The transistor in the center is used as a heating device, while the ones to the left and right serve as temperature sensors. When an airflow passes along the chip from left to right, the left-hand sensing transistor is cooled to a greater degree than its righthand counterpart. This difference is due to the increasing thickness of the laminar boundary layer in the direction of the flow. The temperature difference appears to be proportional to the square root of the flow velocity and changes sign when the flow direction is reversed. Adding another two temperature-sensing transistors above and below the heating transistor makes it feasible to construct a silicon vector flow sensor for measuring flow amplitude and flow direction simultaneously.

\subsection{SMT's for Thermal Signals}

The temperature sensitivity of semiconductor devices is usually considered to be an undesirable property. Yet it can also be put to use to make very accurate temperature sen-

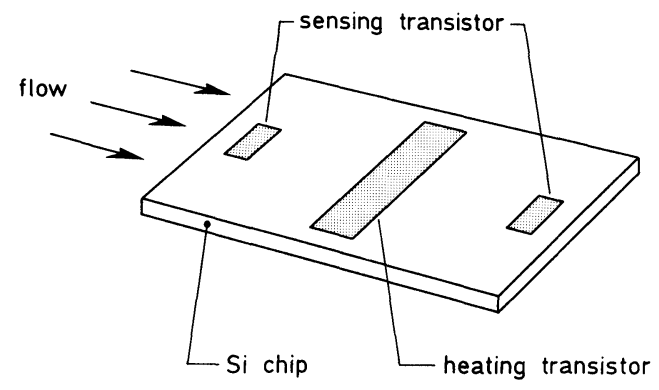

FIGURE 6 Silicon thermoelectric flowmeter. 
sors in a wide variety of applications. Simple diffused resistors can be used as temperature transducers, because the charge-carrier density and the mobility in silicon are a function of the temperature.

Silicon diodes are also suitable as temperature sensors, because the voltage across a forward-biased pn-junction is roughly proportional to the absolute temperature at constant current. However, the best silicon temperature transducers are made with the help of matched transistors. When the transistors are operated at different current densities, the difference in emitter-base voltages is linear to the absolute temperature. Different current densities can be obtained by using identical transistors at two different collector currents, or by using two transistors with unequal emitter areas at equal collector currents.

Devices based on this effect are called PTAT (proportional to absolute temperature) devices. One of their disadvantages is that they usually require three calibration steps for adjusting, e.g. the scale factor. Recently, a new IC design that only needs the calibration of one resistor has been described in the literature. ${ }^{11}$ The device operates between $-50^{\circ} \mathrm{C}$ and $125^{\circ} \mathrm{C}$ with an absolute error of $\pm 0.5^{\circ} \mathrm{C}$ and an excellent long-term stability. By integrating processing circuits on the same chip, devices can be obtained with electronic output signals perfectly suited to interfacing with microprocessors.

\subsection{SMT's for Magnetic Signals}

Magnetic-field measurement equipment contains a magnetic-field sensor whose linearity, range, temperature sensitivity and size are the most important properties. It seems, that silicon lends itself well to constructing sensors with satisfactory characteristics.

Two effects can be employed in silicon: the Hall effect and the magneto-resistance effect. Both effects originate from the fact that an electron or hole moving in a magnetic field experiences the Lorentz force, which is mutually perpendicular to the directions of the particle velocity and the magnetic field. Under influence of the Lorentz force the charge-carriers will "pile-up" at one end of the conductor and charge depletion will occur at the other end. This gives rise to an electrical field equal but opposite in direction to the Lorentz force. The effect in which a voltage is measured perpendicular to the current direction is called the Hall effect. When the Hall voltage is short-circuited by an external conductor or by the current contacts the electric field is reduced to zero and the Lorentz force will deflect the charge-carriers. This results in smaller mobilities and thus in increased resistivity. This effect is referred to the geometrical magneto-resistance effect. Hall plates can easily be fabricated in silicon by diffusing a p-type Hall plate into an ntype epilayer and by providing properly located contacts to the plate. ${ }^{12} \mathrm{~A}$ new and recently described SMT for magnetic fields is based on a two-collector transistor structure in which the current distribution over the collector is modulated by a magnetic field. ${ }^{13}$ As shown in Figure 7 a split buried layer is symmetrically located beneath the emitter base



FIGURE 7 Two-collector structures with split buried layer for measuring magnetic fields. 
of the npn-structure, in order to improve the sensitivity. Electrons leaving the emitter will be deflected under the Hall angle $\theta_{\mathbf{H}}$, which is proportional to the magnetic-field component parallel to the strip emitter and the electron mobility.

The electron deflection causes an unbalance of the collector currents which is proportional to the magnetic field. Linearity within $0.5 \%$ is measured for fields up to $1 \mathrm{~T}$. Based on this sensor a four-collector npn-transistor structure was also developed, which simultaneously measures the X-and Y-components of a magnetic field. The emitter and base in this vector sensor are circular and placed in the center between the four collectors.

\subsection{SMT's for Chemical Signals}

The growing interest in better environmental controls has increased the demand for accurate sensors for chemical measurands. The whole field of analytical chemistry can be represented by a diagram as shown in Figure 8.

Matter can manifest itself in a solid, liquid or gas phase. Moreover, each phase can occur as a perturbance in any other phase, as indicated by the examples given. In order to be able to make full use of the electronic signal-processing systems available today, chemical sensors with similar performance/price ratios and electronic output signals are required.

This challenge has spurred many laboratories on to work on using semiconductors and especially silicon devices for this purpose. However, as past experience has proved, silicon devices are very sensitive to trace impurities and contamination. Therefore, the semiconductor industry has taken great pains to develop passivation and encapsulation techniques to protect the silicon devices and circuits from the environment and to improve their reliability. If silicon is going to be used for chemical sensors, new encapsulation techniques will have to be invented and a very thorough understanding of the silicon measurand interface will have to be provided. These problems have not prevented research groups from entering this difficult field, so that at present, next to the radiantsignal sensors, the chemical-signal field is attracting the most interest. ${ }^{14}$

In the following section the most important SMT's will be discussed.

a) The ISFET. The first chemical SMT was invented in 1968 by Bergveld ${ }^{15}$ and is called an ISFET (ion-sensitive FET). The structure of this device resembles that of a conventional MOSFET, except that no gate electrode is used and that an ion-selective layer $\left(\mathrm{Si}_{3} \mathrm{~N}_{4}, \mathrm{Al}_{2} \mathrm{O}_{3}\right.$, polymers, etc.) is deposited on top of the thermally grown gate oxide layer. Sodium concentration and $\mathrm{pH}$ values have been measured.

b) Hydrogen sensor. When Pd is used in a MOSFET as gate material, it appears to be possible to make a hydrogen sensor. ${ }^{16}$ Its operation seems to be based on the subsequent dissociation of the $\mathrm{H}_{2}$ at the $\mathrm{Pd}$ surface, the diffusion of $\mathrm{H}$ through the Pd layer and the

\begin{tabular}{|c|c|c|c|}
\hline MINOR & GAS & LIQUID & SOLID \\
\hline GAS & $\mathrm{H}_{2}$ IN AIR & $\mathrm{O}_{2}$ IN WATER & $\mathrm{H}_{2}$ IN SmCo \\
\hline LIQUID & FOG & $\begin{array}{l}\text { ALCOHOL } \\
\text { IN } \\
\text { WATER }\end{array}$ & $\begin{array}{c}\text { MOISTURE } \\
\text { IN } \\
\text { PAPER }\end{array}$ \\
\hline SOLID & DUST IN AIR & MILK & ALLOYS \\
\hline
\end{tabular}

FIGURE 8 Matrix showing different combinations of gas, liquid and solid phases. 


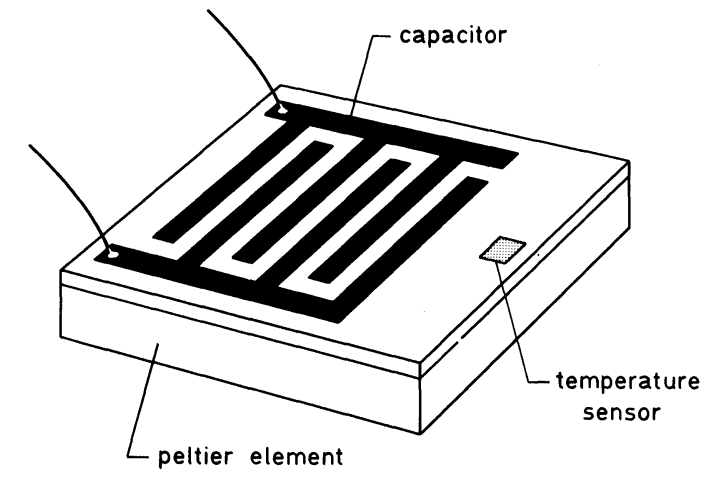

FIGURE 9 Sensor for measuring dew point and humidity.

absorption of $\mathrm{H}$ at the $\mathrm{Pd}-\mathrm{SiO}_{2}$ interface. The Pd work function is changed by this, leading to a change in the threshold voltage of the MOSFET, which can be electronically detected.

c) Humidity sensor. Humidity in air can be accurately measured by a chemical SMT. ${ }^{17}$ The silicon sensor chip consists of a capacitive finger structure for detecting capacitance changes due to dew formation on the chip and a transistor for measuring the chip temperature (Figure 9). The chip is mounted on a miniature Peltier element, which is used as the cooling device. In operation, the temperature of the chip is lowered until water vapor from the air starts to condensate on the chip, and a sharp increase in the capacitance is measured. The temperature at which condensation starts is measured next, giving a temperature from which the relative air humidity can be calculated. The chip size is $2 \times 2 \mathrm{~mm}$, and the width and interspacing of the electrodes are $10 \mu \mathrm{m}$. The non-linearity of the device is less than $0.1 \mathrm{~K}$ over a temperature range of $50 \mathrm{~K}$.

\section{TRANSDUCERS IN THICK- OR THIN-FILM TECHNOLOGY}

Thick- and thin-film technologies have long been used for making electronic circuits, but they can now also be applied to make transducers. Examples of transducers produced by these methods will be given, although we will first turn our attention to some general features.

a) Substrates. The essence of these techniques is to apply patterns to an isolating substrate. A substrate material must not only have the desired electrical properties, but must also be able to resist the process conditions. Examples of materials used are: alumina, beryllia, sapphire and glass. The choice in materials provides an important freedom, so that the mechanical and thermal properties (elasticity, heat conductivity, thermal coefficient of expansion, etc.) can be taken into account.

b) Patterns. Some of the examples given here are based on a specific pattern giving the desired properties. Thin-film technology often makes use of a photolithographic process, while thick-film patterns are made by screen printing. Multilayers can easily be made and add to the versatility of these techniques.

c) Materials. Still other examples get their desired properties mainly by making a choice of the many materials that can be used. A variety of metals, metal oxides, alloys and semiconductors can be evaporated or sputtered and applied as a thin-film. Thick-film pastes are commercially available for conductors, resistors (including ntc and ptc) and die- 




FIGURE 10 Sensor for measuring sunlight power density (Solarimeter) a) frontside with black center and reflecting ring b) backside with thermocouples between center and edge.

lectrics. The formulation of special thick-film pastes has a great potential for adding materials with specified characteristics to a substrate. Passive components will be mostly used, resulting in transducers of the modulating type.

d) Other features. Adding active components to a substrate makes a hybrid circuit. The same process makes it easy to handle electronic signals close to the place where the conversion is accomplished. Adequate potting materials and passivation layers provide a good resistance against environmental circumstances. A hybrid transducer can exhibit a large operating temperature range, mainly as the result of careful material selection and high processing temperatures.

\subsection{Hybrid Transducers for Radiant Signals}

a) Sensor for measuring sunlight power density. A device for measuring the power density of incident sunlight has been realized (Figure 10).

The center of one side of an $\mathrm{Al}_{2} \mathrm{O}_{3}$ substrate is made black. On the other side, the heat of the absorbed sunlight is removed at the edge by a heat sink. The resulting temperature difference between the center and the edge is measured by a series of thermocouples formed by two different thick-film materials. The initial experiments were done with $\mathrm{Au}$ and Pt-Au. The temperature difference is proportional to the power density of the incident sunlight.

b) Sensor for measuring radial electron-beam density. A pattern of concentric electrodes is used to measure the radial electron density of an electron beam. The charge of the incident electrons is coupled to an amplifier by an RC network (Figure 11).

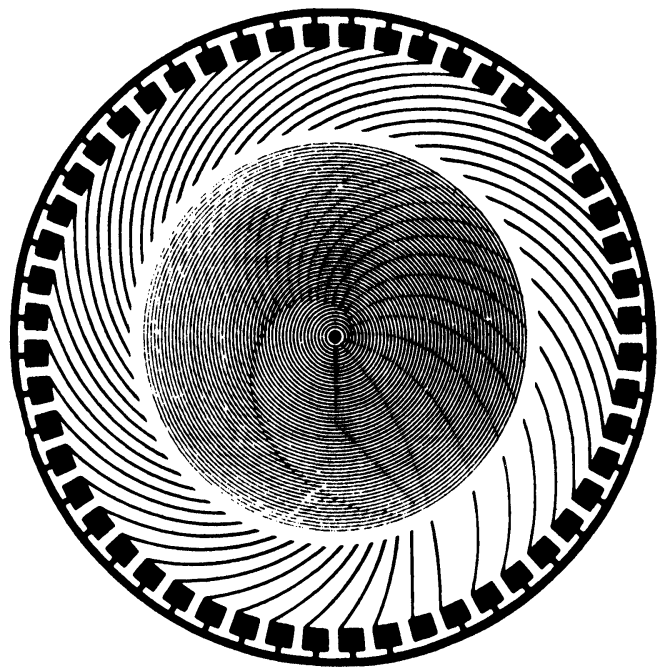

FIGURE 11 Sensor for measuring radial electron beam density. Fifty concentric electrodes connected to resistors at the edge via a dielectric layer. 
c) Light meter. Sputtered or evaporated films of materials such as CdS are used as lightsensitive resistors.

\subsection{Hybrid Transducers for Mechanical Signals}

a) Pressure sensor. The piezoresistive effect in a thick-film resistor is used by Cattaneo, et al. for measuring pressure. ${ }^{18}$ The pressure deforms a ceramic diaphragm on which four thick-film resistors have been deposited in a Wheatstone bridge configuration. The signal is modulated by the displacement of the diaphragm.

b) Strain gauge. Berthe et al. described thin-film strain gauges, in which an isolation layer is first sputtered on a metal substrate.$^{19}$ On top of this a Wheatstone bridge of thinfilm resistors is formed with the aid of a photolithographic process. Thin-film strain gauges can be small and are used for measuring low forces.

c) Displacement sensor. A thin-film microdisplacement transducer has been developed by Klaassen et al. ${ }^{20}$ The transducer consists of two glass plates: the upper is movable and has an electrode capacitivety coupled to the electrodes evaporated, on the lower glass plate. The capacitive coupling is position-dependent. The minimum width and spacing of the electrodes is $100 \mu \mathrm{m}$. In the sensor $90^{\circ}$ phase-shifted sinusoidal sine-wave voltages are used on adjacent fingers of the lower pattern. Distances down to $25 \mathrm{~nm}$ can be detected.

\subsection{Hybrid Transducers for Thermal Signals}

a) Thermocouple. Using thin-film layers of different materials to form a thermocouple is well known. Such a device is very useful in surface temperature measurement, particularly when a fast response is required. Special thick-film pastes consisting of $\mathrm{Pd}-\mathrm{Pt}-\mathrm{Au}$ and $\mathrm{Au}-\mathrm{Pd}$ have been developed for thermocouple use. ${ }^{21}$

b) Thermistor. The formulation and use of a stable thick-film thermistor paste has been reported by Ikegami et al. ${ }^{22}$ The use of this element is illustrated by a number of examples, including the compensation of thermal effects.

c) Temperature sensor. Leppävuori describes a capacitive thick-film temperature sensor making use of the temperature dependence of the $\epsilon_{\mathrm{r}}$ of ferroelectric materials above the Curie temperature. ${ }^{23}$ A mixture of $\mathrm{Ba} \mathrm{Ti}_{3}$ and $\mathrm{Sr} \mathrm{Ti} \mathrm{O}_{3}$ is incorporated in a thick-film paste to serve as an active element.

\subsection{Hybrid Transducers for Magnetic Signals}

Thin-film layers of magnetic material have already been under investigation for a long time. Recently Druyvesteijn reported the application of Ni-Fe thin-films in a read head for a magnetic recorder ${ }^{24}$ By chemical etching a pattern is formed in a $0.05 \mu \mathrm{m}$ thick Ni$\mathrm{Fe}$ film. Conductors are formed on top of it in a sandwich of $\mathrm{Mo}-\mathrm{Au}-\mathrm{Mo}(0.02-0.3-$ $0.05 \mu \mathrm{m})$. Variation in the magnetization of the tape causes the magnetization in the $\mathrm{Ni}$ $\mathrm{Fe}$ film to rotate, which is detected as a variation in the resistance.

\subsection{Hybrid Transducers for Chemical Signals}

a) Moisture sensor. The absorption of water in a porous material causes a change in impedance. Leppävuori reports some experiments concerning the effect of humidity on the conductivity and permittivity of a thick-film capacitor. ${ }^{23}$ Two types of thick-film moisture sensors for permanent installation in highway structures have been described by 
TABLE I

Comparison of technologies for sensors

\begin{tabular}{llll}
\hline Property & Si-Techn. & Thin Film & Thick Film \\
\hline Cost mass production & + & - & 0 \\
Cost small production & - & + & + \\
Reliability encapsulated & + & + & + \\
Reliability non-encapsulated & - & - & 0 \\
Versatility & 0 & 0 & + \\
Electronic compatibility & + & 0 & 0 \\
Possibility of smart sensors & + & - & - \\
Sensor size & + & 0 & 0 \\
Large arrays & 0 & + & + \\
Element density & + & 0 & - \\
Reproducibility & 0 & 0 & 0 \\
Accuracy & 0 & + & + \\
Temperature range & - & + & + \\
Long term stability & + & + & 0 \\
Energy consumption & + & 0 & 0 \\
Handling & - & 0 & + \\
\hline
\end{tabular}

$$
\begin{array}{ll}
\text { Suitability } & + \text { Good } \\
& 0 \text { Moderate } \\
& - \text { Bad }
\end{array}
$$

Lucas et $\mathrm{al}^{25}$ : one using an interdigitated thick-film capacitor as a sensing element, and another consisting of a thick-film heater and thermistor screened and fired on opposite sides of an alumina substrate. With a fixed power input the temperature can be related to the soil-moisture content.

b) $\mathrm{pH}$ sensor. Afromowitz fabricated a $\mathrm{pH}$-sensitive implantable electrode, in which a conductor of Pt-Pd-Au is covered by a pH-sensitive glass layer ${ }^{26}$ Corning 0150 glass, normally used for $\mathrm{pH}$ measurements, is applied via a thick-film paste, thus forming the sensitive film. An impedance conversion is realized on the same substrate.

\section{CONCLUSIONS AND FUTURE OUTLOOK}

Further penetration of micro-electronics into many new markets is hampered by the lack of transducers with performance/price ratios comparable to those of integrated circuits. Silicon planar, thin-film and thick-film technologies all show promise with respect to filling this gap. In Table I the authors have made an attempt to evaluate the suitability of the three technologies for the fabrication of transducers.

This review paper describes a large number of already invented devices, based on these technologies. Because research and development laboratories of industries and universities are focusing their efforts more and more on this burgeoning field, we may be certain that in the next decade many more low-cost and innovative sensors, displays and actuators will follow.

\section{REFERENCES}

1. S. Middelhoek and D.J.W. Noorlag, "Silicon micro-transducers: a new generation of measuring elements" in Modern Electronic Measuring Systems, P.P.L. Regtien ed., (Delft University Press, Delft 1978). 
2. S. Middelhoek, J.B. Angell, D.J.W. Noorlag, "Microprocessors get integrated sensors", IEEE Spectrum (Febr. 1980).

3. S. Middlehoek and D.J.W. Noorlag, "Three-dimensional representation of solid-state transducers", Sensors and Actuators (1981) to be published.

4. S. Middelhoek and D.J.W. Noorlag, "The present and future of silicon micro-transducers", Proc. of Journées d'Electronique et de Microtechnique, Laussanne (1980).

5. D.A. Ross, Optoelectronic Devices and Optical Imaging Techniques, (MacMillan, London 1979).

6. V. Härtel, Optoelectronics, (McGraw-Hill, New York 1978).

7. A. Gieles, "Applications of IC technology in measuring techniques" in Modern Electronic Measuring Systems, P.P.L. Regtien ed. (Delft University Press, Delft 1978).

8. J.B. Angell, "Micro-machined silicon transducer for measuring force, pressure and motion", ESSCIRC 78, Digest of Techn. Papers (1978).

9. D.J.W. Noorlag and S. Middelhoek, "Two-dimensional position-sensitive photodetector with high linearity made with standard IC technology", IEE Journal of Solid State \& Devices, vol. 3 (1979).

10. J.H. Huysing, "Monolithic flow sensors, a survey" in Solid-state Sensors, (Kluwer Deventer 1980).

11. G.C.M. Meyer, "IC temperature transducers with intrinsic reference and their applications in thermocouple cold-junction compensation" in Solid-state Sensors, (Kluwer, Deventer 1980).

12. J.E.L. Hollis, "Micro-electronic magnetic transducers", Meas. and Control, vol. 6 (1973).

13. V. Zieren, "A new silicon micro-transducer for the measurement of the magnitude and direction of a magnetic-field vector", Proc. IEDM, Washington (1980).

14. J.N. Zemel, "Semiconductor physics of surface devices" in Proc. Nato School on Chemically Sensitive Electronic Devices, Sensors and Actuators, (1981).

15. P. Bergveld and N.F. de Rooij, "The single electrode operation of an ISFET" in Theory, Design and Biomedical Applications of Solid-state Chemical Sensors", (CRS Press 1978).

16. K.I. Lundström, M.S. Shivaraman, C.H. Svensson, "A hydrogen-sensitive Pd-gate MOS transistor", J. Appl. Phys., vol. 46 (1975).

17. P.P.L. Regtien, "An integrated humidity sensor" in Solid-state Sensors, (Kluwer, Deventer 1980).

18. A. Cattaneo, R. DEll Acqua, F. Forlani and L. Pirozzi, "The industrial application of the piezoresistive effect: a low-cost thick-film pressure sensor", Proc. Electronic Components Conference, San Francisco (1980).

19. K. Bethe and D. Schön, "Thin-film strain gauge transducers", Philips Techn. Review 39 (1980).

20. K.B. Klaassen, J.C.L. van Peppen and R.E. Sandbergen, "Thin-film microdisplacement transducer", Proc. Eurocon Stuttgard 1980, in Electronics to Micro-electronics, W.A. Kaiser and W.E. Proebster ed., (North Holland Publishing Co. 1980).

21. A.R. Robertson, "Thick-film thermocouples - A new approach to temperature measurement", Proc. Electronic Components Conference, San Francisco (1976).

22. A. Ikegami, H. Arima, H. Tosaki, Y. Matsuoka, M. Ai, H. Minorikawa and Y. Asákino, "Thickfilm thermistor and its applications", Proc. Electronic Components Conference, San Francisco (1980).

23. S. Leppävuori, "New thick-film sensors", European Hybrid Micro-electronics Conference, Ghent (1979).

24. W.F. Druyvesteijn, "Magneto-resistive sensor", in Solid-state Sensors, (Kluwer, Deventer 1980).

25. M.S.P. Lucas, M.R. Casey, R.K. Blockstome and W.H. Dawes, "Thick-film moisture sensors", Proc. Electronic Components Conference, Washington D.C. (1974).

26. M.A. Afromowitz and S.S. Yee, "Fabrication of pH-sensitive implantable electrode by thickfilm hybrid technology", Proc. Thin- and Thick-film Technology Conference, Augsburg (1977), (VDE-Verlag, Berlin 1977). 

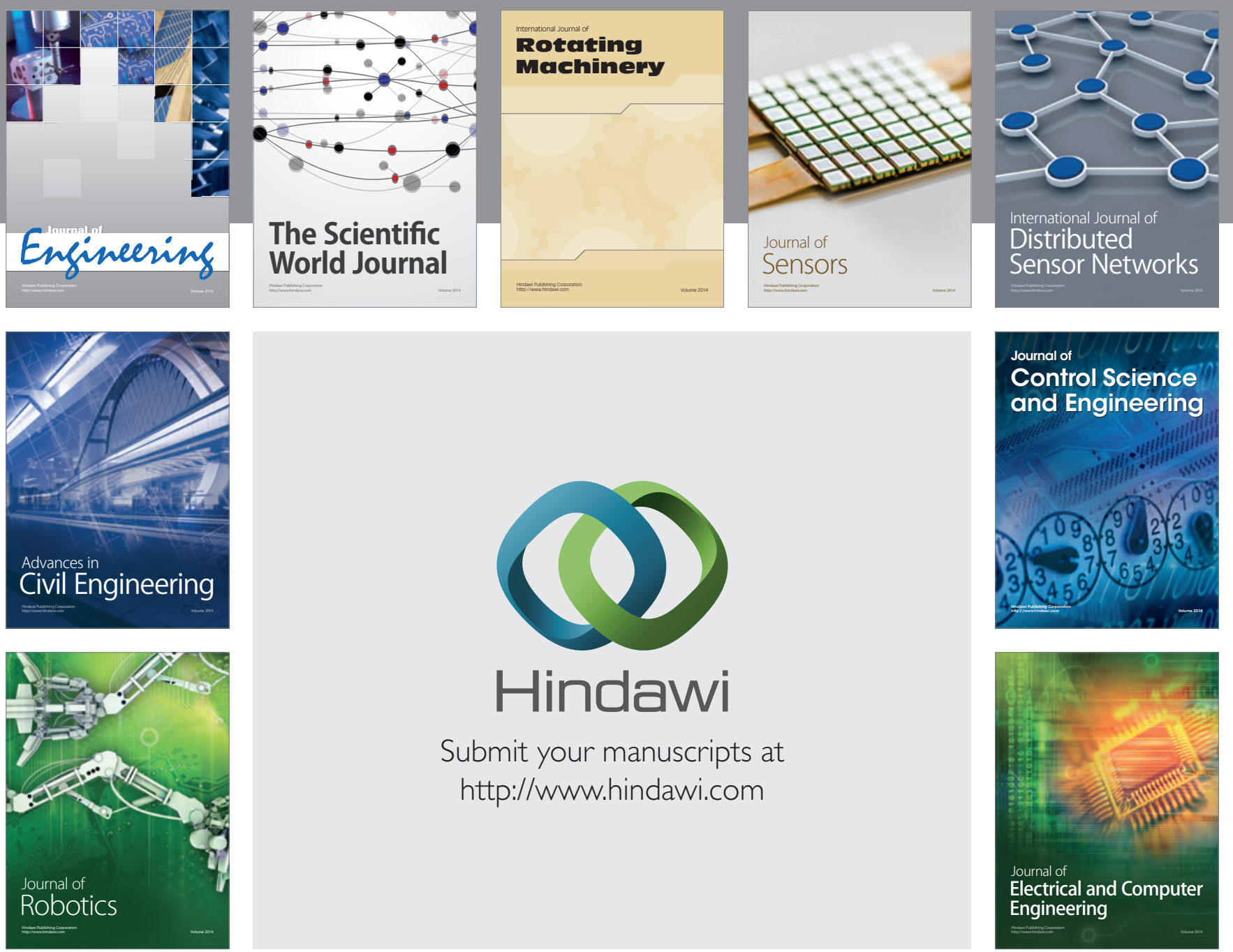

Submit your manuscripts at

http://www.hindawi.com
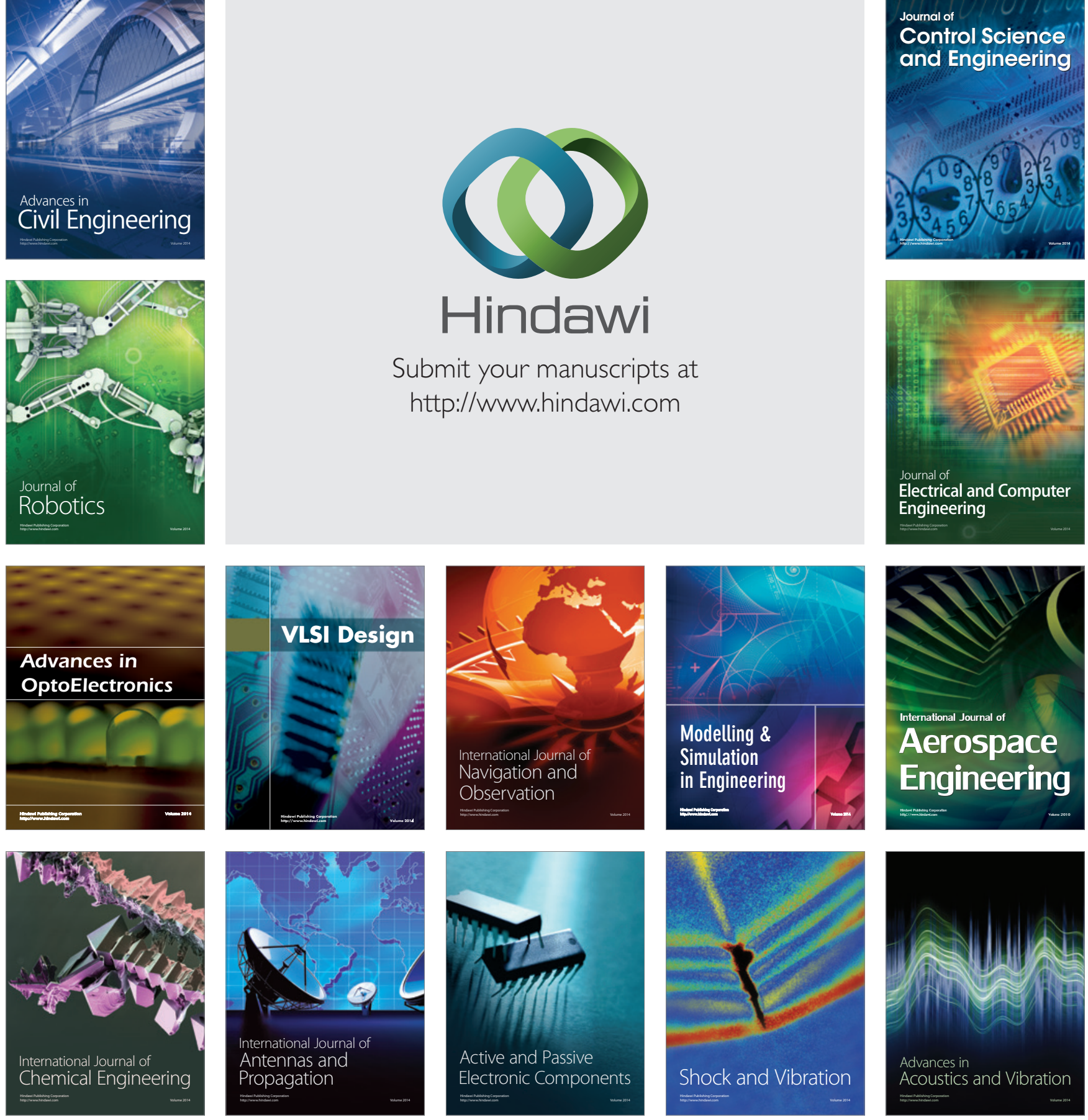